\title{
BMJ Open Efficacy and safety of anti-epidermal growth factor receptor agents for the treatment of oesophageal cancer: a systematic review and meta-analysis
}

\author{
Lijuan Zhang, ${ }^{1}$ Yanli Song, ${ }^{1}$ Nan Jiang, ${ }^{2}$ Yaqi Huang, ${ }^{2}$ Bo Dong, ${ }^{1}$ Wei Li, ${ }^{1}$ \\ Yanze He, ${ }^{1}$ Yun Chen, ${ }^{3}$ Haibin Liu, ${ }^{4}$ Rui Yu (iD ${ }^{5}$
}

To cite: Zhang L, Song Y, Jiang $\mathrm{N}$, et al. Efficacy and safety of anti-epidermal growth factor receptor agents for the treatment of oesophageal cancer: a systematic review and meta-analysis. BMJ Open 2021;11:e046352. doi:10.1136/ bmjopen-2020-046352

- Prepublication history and additional material for this paper are available online. To view these files, please visit the journal online (http://dx.doi. org/10.1136/bmjopen-2020046352).

LZ, YS and NJ contributed equally.

Received 28 0ctober 2020 Revised 25 February 2021 Accepted 03 March 2021

Check for updates

(C) Author(s) (or their employer(s)) 2021. Re-use permitted under CC BY-NC. No commercial re-use. See rights and permissions. Published by BMJ.

For numbered affiliations see end of article.

Correspondence to

Dr Rui Yu; yurui1969@163.com

\section{ABSTRACT}

Objectives Despite remarkable advances in the treatment of oesophageal cancer $(\mathrm{OC})$, the role of antiepidermal growth factor receptor (anti-EGFR) agents in treating OC remains controversial. Herein, a systematic review and meta-analysis were conducted to elucidate the efficacy and safety of anti-EGFR agents in patients with OC. Design Meta-analysis of randomised controlled trials (RCTs) identified by searching the PubMed, Embase, Web of Science, ClinicalTrials.gov, Cochrane Library, Chinese Biology Medicine, China National Knowledge Infrastructure and Wanfang Data Knowledge Service Platform databases from inception to December 2019. We followed the Preferred Reporting Items for Systematic Reviews and Meta-Analyses guidelines.

Setting RCTs from any country and healthcare setting. Participants Patients with OC. Interventions Combination therapy with anti-EGFR agents and conventional treatments versus conventional treatments alone in patients with $\mathrm{OC}$.

Primary and secondary outcome measures Overall survival (OS) and progression-free survival (PFS) were primary outcome measures, and objective response rate (ORR), disease control rate (DCR) and treatment toxicities were secondary outcome measures.

Results In total, 25 RCTs comprising 3406 patients with $\mathrm{OC}$ were included. Overall, anti-EGFR treatment significantly improved the OS (HR: $0.81,95 \% \mathrm{Cl} 0.74$ to $0.89, \mathrm{p}<0.00001$ ), ORR (relative risk (RR): $1.33,95 \% \mathrm{Cl}$ 1.16 to $1.52, \mathrm{p}<0.0001)$ and DCR (RR: $1.22,95 \% \mathrm{Cl} 1.11$ to $1.34, p<0.0001$ ) but not PFS (HR: $0.91,95 \% \mathrm{Cl} 0.76$ to $1.08, p=0.26)$. Anti-EGFR treatment was significantly associated with higher incidences of myelosuppression, diarrhoea, acne-like rash and hypomagnesaemia. Conclusions Overall, anti-EGFR agents have positive effects on OS, the ORR and DCR in OC. However, considering the high incidence of adverse effects, such as myelosuppression, diarrhoea, acne-like rashes and hypomagnesaemia, careful monitoring of patients with $\mathrm{OC}$ is recommended during anti-EGFR treatment. Trial registration number CRD42020169230.

\section{INTRODUCTION}

Oesophageal cancer (OC) is the seventh most common cancer type and the sixth
Strengths and limitations of this study

- This meta-analysis is the first attempt to summarise the evidence of the efficacy and safety of anti-EGFR agents in combination with conventional treatments versus conventional treatments alone for oesophageal cancer.

- Eight databases, a manual search and unpublished studies were retrieved to identify the eligible studies.

- Quality of the eligible studies was assessed using the Cochrane Handbook for Systematic Reviews and Meta-Analysis.

- This study has been registered a priori and followed the Preferred Reporting Items for Systematic Reviews and Meta-Analyses guidelines.

- Publication, reporting or selection bias cannot be ruled out because of the lack of access to individual patient data.

leading cause of cancer-related mortality, accounting for approximately 1 in 20 cancerrelated deaths worldwide in 2018. ${ }^{1}$ However, the incidences of $\mathrm{OC}$ and mortality rates vary considerably, as demonstrated by a twofold to threefold difference in statistical data between different geographical regions. ${ }^{2} \mathrm{OC}$ is common in Eastern and Southern Africa, but the highest incidence rates have been reported in East Asia, with those in Mongolia and China being among the top five worldwide. ${ }^{12}$ These highest risk areas are called the 'oesophageal cancer belt', with squamous cell cancers showing a dominance of $90 \%$, while adenocarcinoma being predominant in the people of European descent. ${ }^{1}$

OC is diagnosed in later stages in $80 \%$ of the patients, and typically, few patients benefit from single-modality treatments, such as surgery (5-year overall survival (OS) of 15\%-20\%), chemotherapy (CT; 5-year OS of $15 \%-45 \%)$ and radiotherapy (RT; 5 -year OS of $0 \%-15 \%) .{ }^{3}$ Therefore, chemoradiotherapy 
(CRT; 5-year OS of 26\%) has become a standard of care for OC. However, controlling locoregional recurrence or persistent disease remains a major challenge. ${ }^{4}$ The use of higher radiation doses, combination of various CT regimens and RT or other combination therapies (eg, surgery and neoadjuvant CT) have failed to achieve any improvement. ${ }^{5-8}$ Therefore, the addition of novel therapies to conventional single or multimodality treatments is urgently needed.

The epidermal growth factor receptor (EGFR) signalling pathway is implicated in the proliferation, invasion, angiogenesis and survival of cancer cells. ${ }^{9}$ Moreover, overexpression of the EGFR in OC has been associated with a poor prognosis. ${ }^{10}$ Previous studies have shown synergistic effects between EGFR inhibitors and conventional treatments. ${ }^{11}{ }^{12}$ Hence, there is a strong rationale for testing EGFR-targeting drugs to design an effective treatment strategy against OC. Tyrosine kinase inhibitors (TKIs) and monoclonal antibodies (mAbs) are two promising classes of anti-EGFR drugs. mAbs bind to the extracellular domain of the EGFR and compete with endogenous ligands to block the ligand-binding region and ligandinduced EGFR tyrosine kinase activation. ${ }^{13}$ TKIs reversibly compete with adenosine 5' triphosphate (ATP) to bind to the intracellular catalytic domain of EGFR tyrosine kinase and inhibit EGFR autophosphorylation and downstream signalling. ${ }^{13}$ Various anti-EGFR agents, such as cetuximab, ${ }^{1415}$ nimotuzumab, ${ }^{1617}$ panitumumab, ${ }^{18}$ erlotinib $^{19} 20$ and gefitinib, ${ }^{21} 22$ are used for treating OC. However, the role of anti-EGFR agents in combination with conventional treatments is controversial, and toxicity associated with these agents is variable and uncertain. ${ }^{14-17}$ 19-22 Moreover, there is no clear evidence that combination of anti-EGFR agents and conventional treatments is superior to conventional treatments alone for OC. To address this research gap, we conducted a systematic review and meta-analysis of randomised controlled trials (RCTs) to evaluate the efficacy and safety of anti-EGFR agents in combination with conventional treatments versus conventional treatments alone in patients with OC.

\section{METHODS}

\section{Literature search strategy}

A literature search of the following databases (from inception to December 2019) was performed by two authors to identify eligible studies: PubMed, Embase, Web of Science, ClinicalTrials.gov, the Cochrane Library, Chinese Biology Medicine, China National Knowledge Infrastructure and the Wanfang Data Knowledge Service Platform. Furthermore, a manual search using the reference lists of all the retrieved articles and relevant journals was conducted to identify additional eligible studies. Unpublished studies were retrieved from the System for Information on Grey Literature database to reduce publication bias. Combinations of Medical Subject Headings/ Emtree terms and free-text terms were used to search the above databases. The location of the primary OC site included typical endoscopic measurements of each region from the incisors (range: from 15 to $42 \mathrm{~cm}$ ), starting from the upper oesophageal sphincter to the proximal $2 \mathrm{~cm}$ of the cardia. ${ }^{23}$ The following main search terms were used: 'oesophageal neoplasms', 'epidermal growth factor receptor inhibitor', 'cetuximab', 'panitumumab', 'nimotuzumab', 'erlotinib', 'gefitinib', 'randomised controlled trial' and 'clinical trial'. The full search strategies used are presented in online supplemental data S1.

Two authors independently screened the studies based on the selection criteria. Initially, each title and abstract were screened, and the full text of the eligible studies was retrieved. Any disagreements between the two authors during the selection process were settled by discussion and consensus with a third author.

\section{Inclusion and exclusion criteria}

Eligible studies were included if they met the following criteria: (1) patients with histologically confirmed OC; (2) anti-EGFR treatment versus conventional treatment (defined as any single- or multimodality treatment for OC, such as surgery, CT, RT or any combination of these); (3) RCT; and (4) HRs with 95\% CIs reported for OS and/or progression-free survival (PFS), or sufficient data provided to calculate these values.

Repeated studies, reviews, comments, letters, case reports, meeting abstracts, study protocols or animal studies were excluded. Duplicate and incomplete data were also excluded.

\section{Data extraction and quality assessment}

Data extraction and quality assessment were carried out by two independent authors, and discrepancies were resolved by discussion and consensus with the third author.

The extracted information for each eligible study included the name of the first author, publication year, country, study design, enrolment period, sample size, mean/median age of patients (range, years), treatment protocol and major clinical outcomes (OS, PFS, objective response rate (ORR), disease control rate (DCR) and grade $3-5$ toxicities).

The quality of each eligible study was assessed using the Cochrane Handbook for Systematic Reviews of Interventions V.6.0 (www.training.cochrane.org/handbook). Random sequence generation, allocation concealment, blinding, incomplete outcome data and selective outcome reporting were examined to categorise each eligible study as having a: (A) low, (B) moderate or (C) high risk of bias.

\section{Statistical analysis}

The primary outcomes were OS and PFS, expressed as the HR with a $95 \% \mathrm{CI}$, which were calculated from the number of observed deaths minus the number of estimated deaths and its variance. ${ }^{24} \mathrm{OS}$ was defined as the time from diagnosis to death or to the last follow-up, and PFS was defined as the time from diagnosis to the first documented recurrence. Both OS and PFS data were obtained 
from Kaplan-Meier curves using Engauge Digitizer V.10.8 (http://sourceforge.net/projects/digitizer/). Secondary outcomes, including ORR, DCR and haematological and non-haematological toxicities, were expressed as relative risk (RR) ratios with 95\% CIs using the Mantel-Haenszel method. ORR was defined as the rate of partial response (PR) and complete response (CR), and DCR was defined as the rate of $\mathrm{PR}, \mathrm{CR}$ and stabilisation.

Statistical heterogeneity across studies was evaluated using the $\chi^{2}$ test, with a level of significance set at $\mathrm{p}<0.1$, and quantified with the $I^{2}$ statistic. The fixed-effects model was used if the heterogeneity test showed no statistical significance $\left(I^{2}<50 \% ; \mathrm{p}>0.1\right)$; otherwise, the randomeffects model was adopted. Publication bias was assessed by inspecting funnel plots for asymmetries and outliers. Sensitivity analysis was conducted to explore the robustness of the results by excluding certain studies to recalculate the pooled HR and RR estimates. Five studies were excluded because of small sample sizes or treatments with surgery and/or induction CT. ${ }^{14}{ }^{2025-27}$ A subgroup analysis was performed based on the various treatment regimens.

All analyses were conducted using Review Manager V.5.3 (Cochrane Collaboration, Oxford, UK). A twosided $p$ value $<0.05$ was considered significant for all analyses, except heterogeneity tests. This study was reported according to the Preferred Reporting Items for Systematic Reviews and Meta-Analyses guidelines. ${ }^{28}$

\section{Patient and public involvement}

Patients and/or the public were not involved in the design, conduct, reporting or dissemination plans of this research.

\section{RESULTS \\ Study selection}

A total of 1374 records were identified by searching the databases, and no additional records were found using other sources. After removing duplicates, 1298 records remained. Based on the screening of the titles, 952 records were excluded as not meeting the eligibility criteria. Next, the remaining 346 abstracts were further evaluated, of which 301 records were excluded as they were reviews $(n=114)$, non-RCTs $(n=108$, including case reports, meeting abstracts, letters, editorials and news), animal studies $(n=3)$, or studies on other cancers and drugs $(n=76)$ unrelated to this study. After screening the remaining 45 full-text articles, 20 records were excluded as they were non-RCTs $(n=5)$, ongoing studies $(n=2)$, studies on other cancers and drugs $(n=8)$ or studies without outcomes $(n=5)$. Finally, 25 RCTs were included in the quantitative synthesis for this metaanalysis. The flow diagram of the selection process is shown in figure 1.

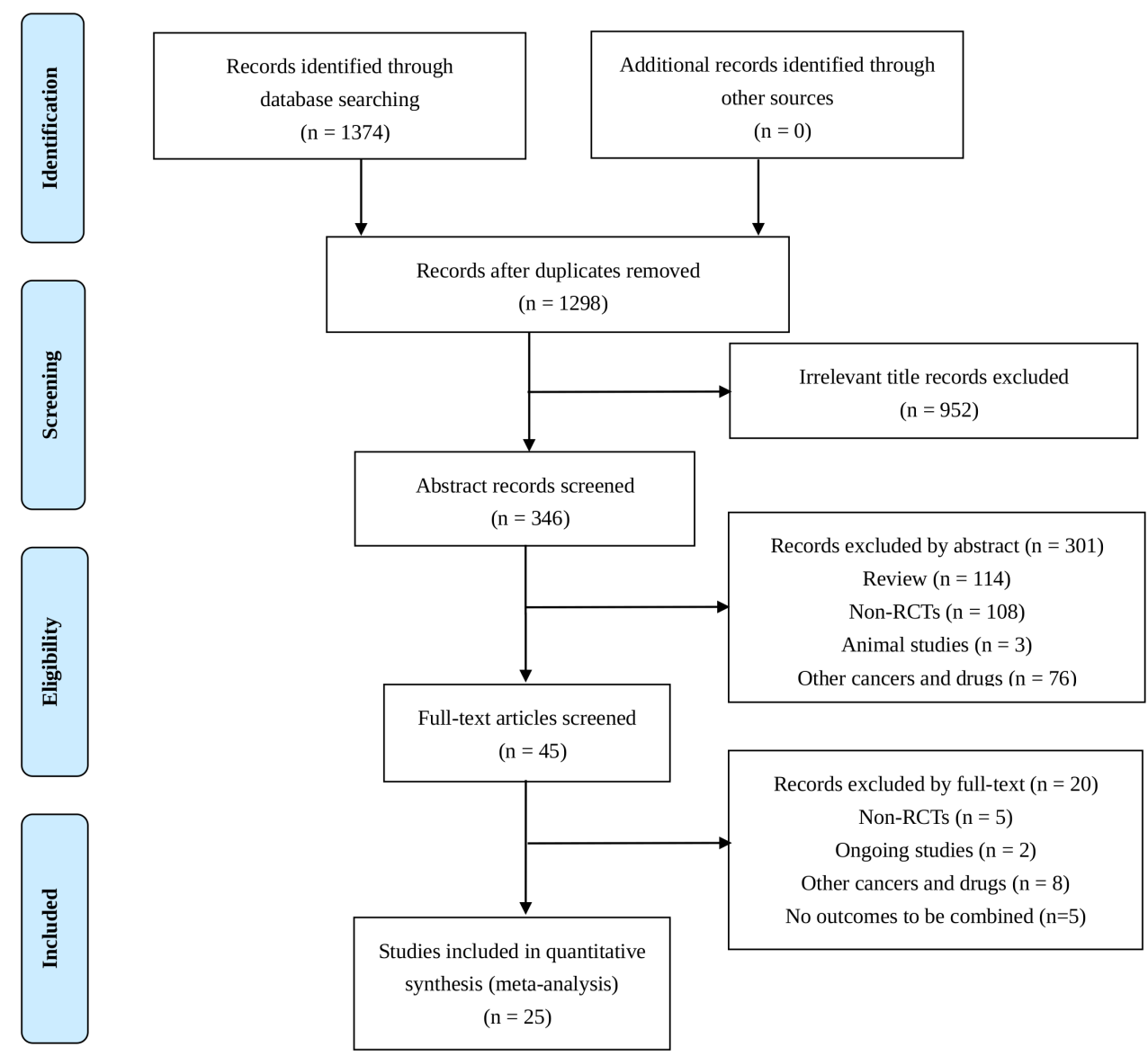

Figure 1 Flow diagram of the selection process of relevant studies. RCTs, randomised controlled trials. 


\section{Study characteristics}

The eligible studies were published between 2009 and 2018, and a total of 3406 patients were included in this meta-analysis. The sample sizes of the studies ranged from 16 to 225 patients. In total, 1699 patients were in the anti-EGFR arm and 1707 patients were in the nonanti-EGFR arm. The age of the patients ranged from 26 to 87 years. Seventeen studies were conducted in China, ${ }^{19-21}$ 25-27 29-39 three in the $\mathrm{UK}^{22} 4041$ and one each in the USA, ${ }^{15}$ Germany, ${ }^{11}$ Switzerland, ${ }^{14} \mathrm{Cuba}^{17}$ and Brazil. ${ }^{16}$ Two studies from the UK were performed on the same group of patients, and the study periods partly overlapped. ${ }^{40}$ However, each study analysed different outcomes. In the anti-EGFR arms, the patients were administered anti-EGFR agents, including cetuximab, nimotuzumab, erlotinib or gefitinib, in combination with surgery, CT, RT or CRT. In the non-anti-EGFR arms, the patients underwent only surgery, CT, RT or CRT. The basic characteristics of the 25 selected studies are presented in online supplemental table S1.

\section{Quality assessment}

The quality assessment data for each study are shown in table 1 . Briefly, among the 25 RCTs, 1 study was rated as level A, ${ }^{22} 17$ studies as level B ${ }^{15202125-2729-39}$ and 7 studies as level C. ${ }^{1} 141617194041$ Eleven of the included studies were assessed to have a low risk of random sequence generation, ${ }^{14-16} 222931$ 37-41 and six studies used allocation concealment. ${ }^{11} 1416224041$ Only one study reported the blinding of participants and outcome assessment. ${ }^{22}$ All the studies were assessed to have a low risk of bias because they addressed incomplete outcome data and selective reporting. No other source of bias was identified in these studies.

\section{Efficacy analysis}

Fifteen studies ${ }^{11}$ 14-16 19222630323335 37-40 were included in the analysis of OS, which showed significant improvement in the anti-EGFR arm (HR: $0.81,95 \%$ CI 0.74 to $0.89, \mathrm{p}<0.00001 ; I^{2}=40 \%, \mathrm{p}=0.05$ ) (figure 2). A subgroup analysis indicated that the administration of anti-EGFR agents in combination with CT, CRT or RT improved the OS of patients with OC. Both anti-EGFR mAbs and TKIs, especially nimotuzumab and erlotinib, significantly improved the OS (online supplemental figure S1).

Six studies ${ }^{111422} 263640$ were included in the evaluation of PFS, which demonstrated no significant difference between the anti-EGFR and control arms (HR: 0.91, 95\% CI 0.76 to $1.08, \mathrm{p}=0.26 ; I^{2}=53 \%, \mathrm{p}=0.06$ ) (figure 3 ). A longer PFS was observed in patients treated with nimotuzumab, but no significant differences were found for other treatments (online supplemental figure S2).

Analysis of 16 studies ${ }^{11} 1720212627$ 29-31 33-39 that evaluated the ORR indicated that the ORR significantly increased in the anti-EGFR arm (RR: 1.33, 95\% CI 1.16 to $1.52, \mathrm{p}<0.0001 ; I^{2}=70 \%, \mathrm{p}<0.0001$ ) (online supplemental figure S3). A higher ORR was reported in patients with OC who received anti-EGFR agents in combination with CT, CRT, surgery or RT. Both antiEGFR mAbs and TKIs, especially nimotuzumab and erlotinib, significantly increased the ORR (online supplemental figure $\mathrm{S} 4$ ).

Fifteen studies ${ }^{11} 17202126{ }^{29-38}$ that analysed the DCR showed that the DCR was significantly improved in the anti-EGFR arm (RR: $1.22,95 \%$ CI 1.11 to $1.34, \mathrm{p}<0.0001$; $I^{2}=70 \%, \mathrm{p}<0.0001$ ) (online supplemental figure S5). Combination treatment with anti-EGFR agents and CT or RT resulted in a higher DCR. Anti-EGFR mAbs and TKIs, particularly cetuximab and erlotinib, decreased the risk of DCR (online supplemental figure S6).

\section{Safety analysis}

Six types of haematological toxicities were analysed in this meta-analysis, including anaemia, leucopenia, neutropaenia, thrombocytopaenia, lymphopaenia and myelosuppression. The results of the pooled analysis demonstrated that there was no significant association between anti-EGFR agents and the increased risk of haematological toxicities, except for myelosuppression (RR: $0.42,95 \%$ CI 0.21 to $0.86, \mathrm{p}=0.02$ ) (online supplemental figure S7). A subgroup analysis revealed that the addition of anti-EGFR agents to CT was associated with an increased risk of myelosuppression compared with that of CT alone, whereas no significant difference was found in the case of CRT. Additionally, among patients who received anti-EGFR mAbs, only those treated with nimotuzumab displayed a significant difference in the incidence of myelosuppression (RR: $0.39,95 \%$ CI 0.18 to $0.83, \mathrm{p}=0.02$ ) (online supplemental figure $\mathrm{S} 8$ ).

Eight non-haematological toxicities were reported, namely cardiac toxicity, gastrointestinal toxicity, dermatological toxicity, pulmonary toxicity, renal toxicity, constitutional symptoms, infection and laboratory findings. The results indicated statistically significant differences for dermatologic toxicity (RR: $9.83,95 \%$ CI 3.54 to 27.28 , $\mathrm{p}<0.0001)$ and laboratory findings (RR: $1.59,95 \%$ CI 1.05 to $2.42, \mathrm{p}=0.03$ ) in the anti-EGFR arm, whereas no significant differences were observed for other nonhaematological toxicities (online supplemental table S2). A subgroup analysis confirmed that patients who received anti-EGFR treatment had higher incidences of diarrhoea, acne-like rashes and hypomagnesaemia than those in the control group (online supplemental table S2). Higher incidences of diarrhoea, acne-like rashes and hypomagnesaemia were observed in patients who received anti-EGFR agents in combination with CRT than in those who received CRT alone (online supplemental figures S9-S11). Increased risks of diarrhoea, acne-like rashes and hypomagnesaemia were found among patients who received antiEGFR mAbs, especially cetuximab (online supplemental figures S9-S11). Additionally, anti-EGFR TKIs, particularly erlotinib, increased the incidence of acne-like rash (online supplemental figure S10), whereas gefitinib was associated with an increased risk of diarrhoea (online supplemental figure S9). 


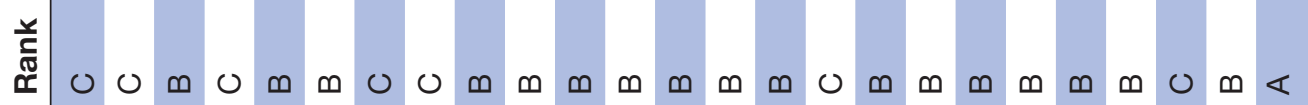

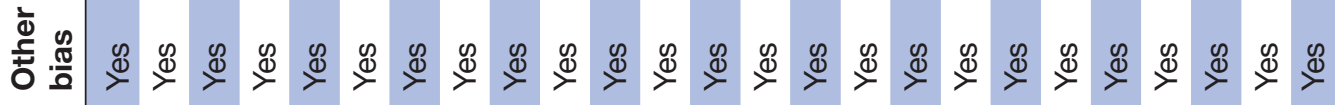

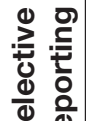

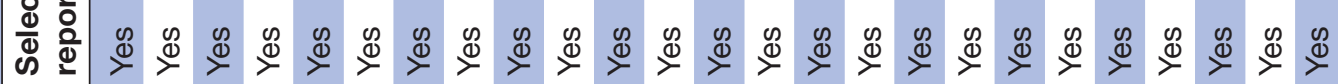

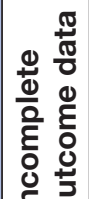

主 $\stackrel{Ð}{\check{c}}$

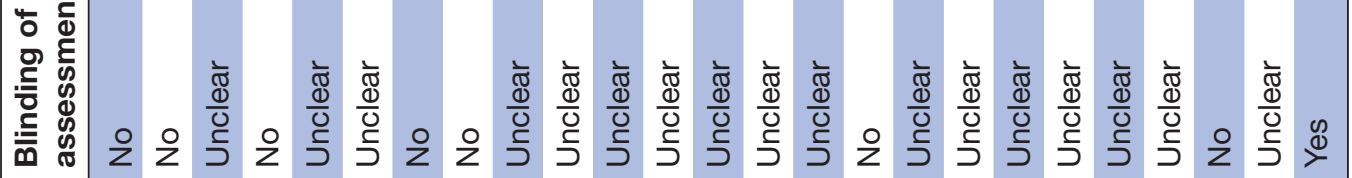

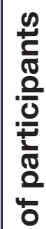

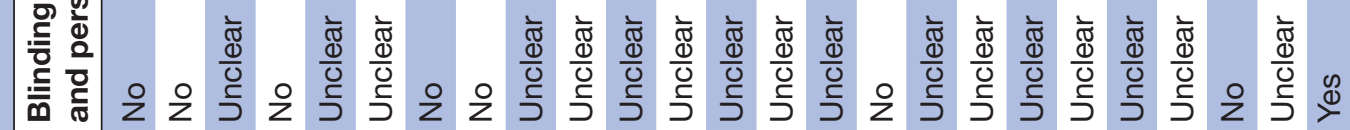

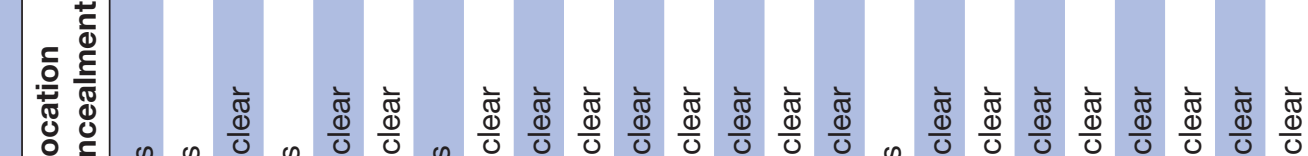

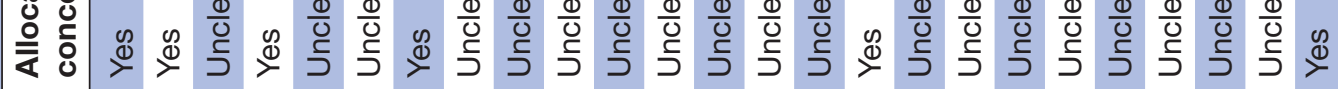

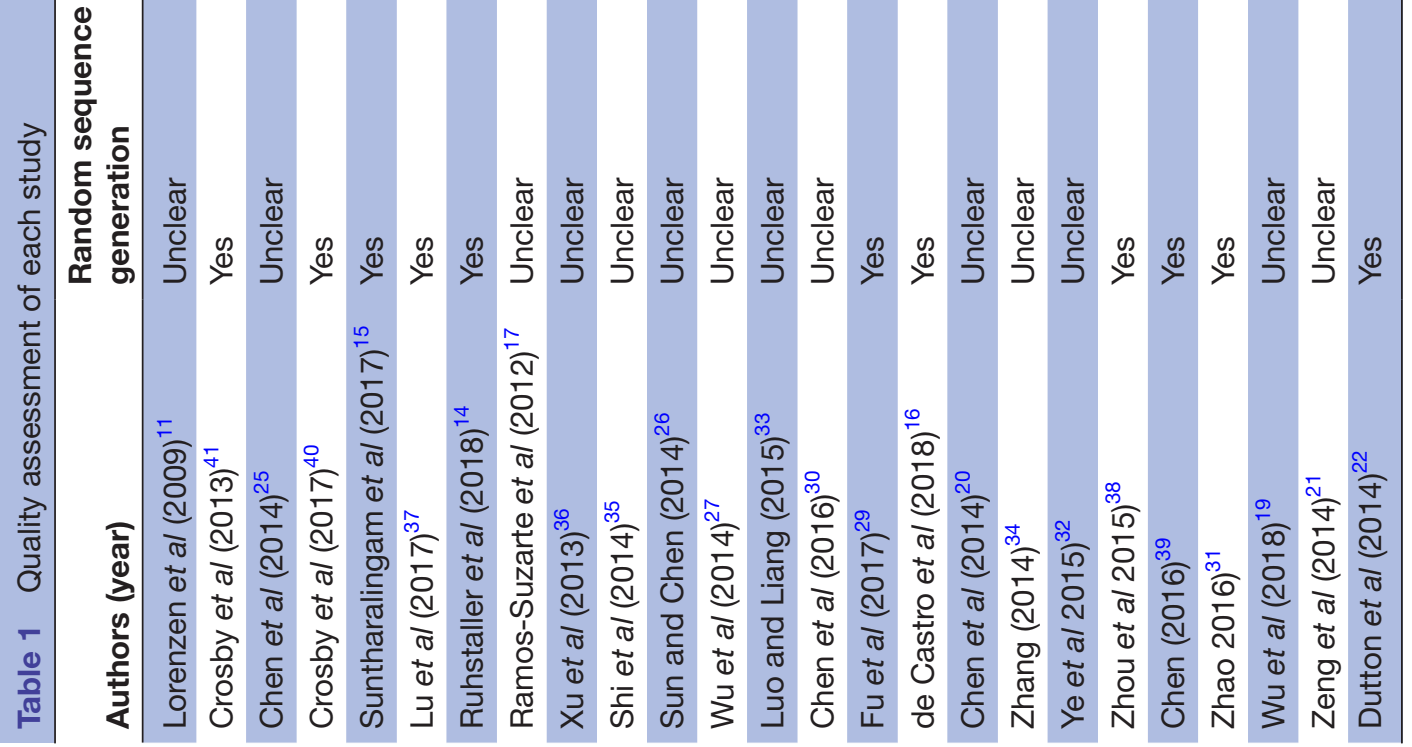

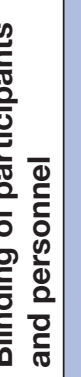




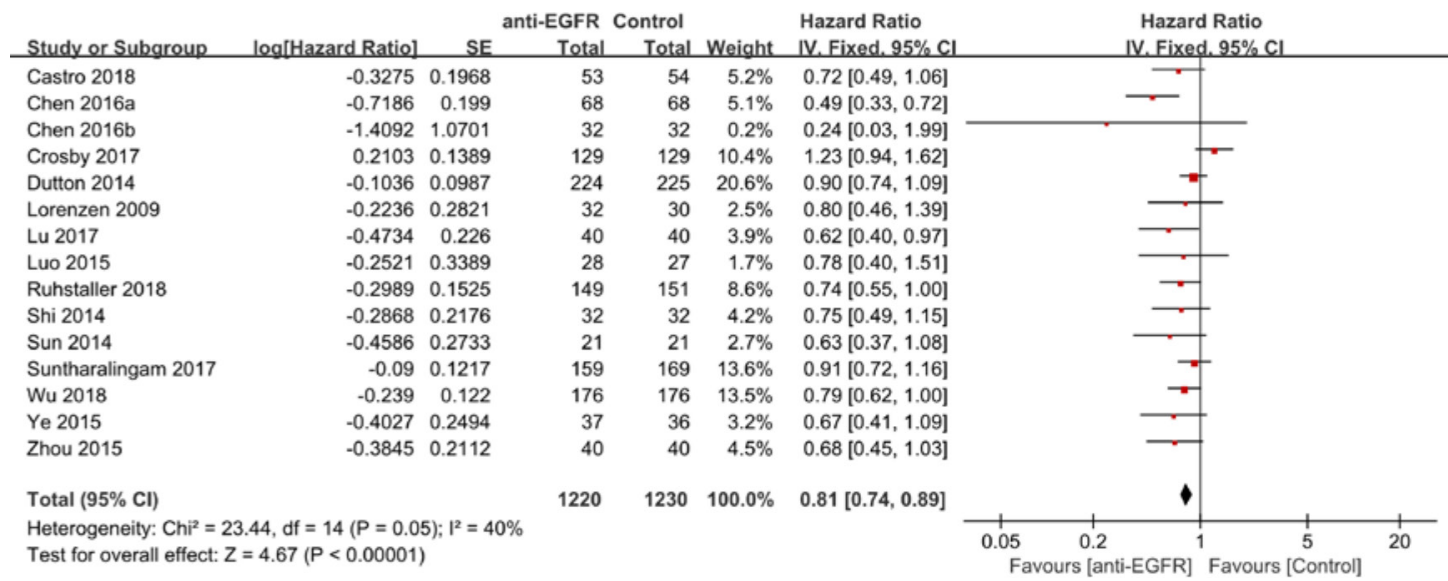

Figure 2 Forest plot of the overall survival of patients with oesophageal cancer in the anti-EGFR arm versus the non-antiEGFR arm. Anti-EGFR, antiepidermal growth factor receptor.

\section{Sensitivity analysis}

For sensitivity analysis, studies by Ruhstaller $e t a l,{ }^{14}$ Chen $e t$ $a l,{ }^{25}$ Chen $e t a l,{ }^{20}$ Sun and $\mathrm{Chen}^{26}$ and Wu et al $2014^{27}$ were excluded. No significant changes in the pooled estimates of OS, PFS, ORR and DCR indicated that the results were statistically stable and reliable (online supplemental table S3). No other sensitivity analysis had any effect on the results.

\section{Publication bias}

A funnel plot of the included studies, which was used to assess the possibility of publication bias, showed an asymmetry, thereby indicating that the publication bias existed (figure 4, online supplemental figures S12, and S13).

\section{DISCUSSION}

OC, a malignant digestive system neoplasm with a poor prognosis, is regarded as a threat to public health. Currently, anti-EGFR agents are commonly used as valuable therapeutic options for OC. However, various studies have published contradictory results regarding these agents. Therefore, we conducted this meta-analysis of RCTs to evaluate the efficacy and safety of combination therapy with anti-EGFR agents and conventional treatments compared with those of conventional treatments alone. To the best of our knowledge, this is the first meta-analysis to evaluate the benefits and toxicities of anti-EGFR and non-anti-EGFR treatment strategies for OC. The findings of this meta-analysis indicated that
anti-EGFR including treatments might be more effective than non-anti-EGFR treatments in terms of prolonging the OS and increasing the ORR and DCR in patients with OC. However, anti-EGFR agents may be associated with higher incidences of myelosuppression, diarrhoea, acnelike rashes and hypomagnesaemia.

The addition of anti-EGFR agents to CT, CRT or RT regimens significantly improved the OS of patients with OC. Because CRT is the standard treatment approach for OC, the combination of anti-EGFR agents with CT or RT was not considered in this meta-analysis, owing to limited availability of studies. According to this meta-analysis, the addition of anti-EGFR agents to CRT may be an important strategy to improve clinical outcomes in patients with OC. Most importantly, anti-EGFR mAbs and TKIs, especially nimotuzumab and erlotinib, significantly improved the OS. The clinical benefits of nimotuzumab may be explained by its ability to directly inhibit the EGFR signalling pathway, which results in an antiproliferative effect on tumour cells. ${ }^{42}$ Furthermore, nimotuzumab enhances the sensitivity of tumour cells to RT and $\mathrm{CT},{ }^{43}$ which may explain the positive results obtained in this study. The combination of erlotinib with conventional treatments for OC is still controversial because of the limited number of RCTs that tested this TKI. TKIs have been demonstrated to induce G1/S phase cell cycle arrest in various cancers. Studies have also reported that TKIs inhibit the growth of OC cells, ${ }^{44-47}$ which might explain the significant improvement of OS in the present study. However,

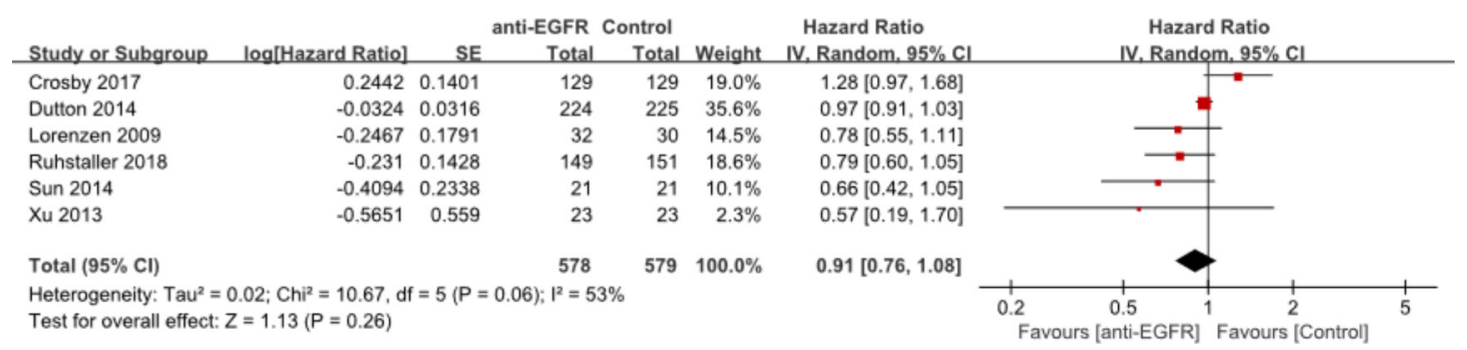

Figure 3 Forest plot of the progression-free survival of patients with oesophageal cancer in the anti-EGFR arm versus the nonanti-EGFR arm. Anti-EGFR, antiepidermal growth factor receptor. 


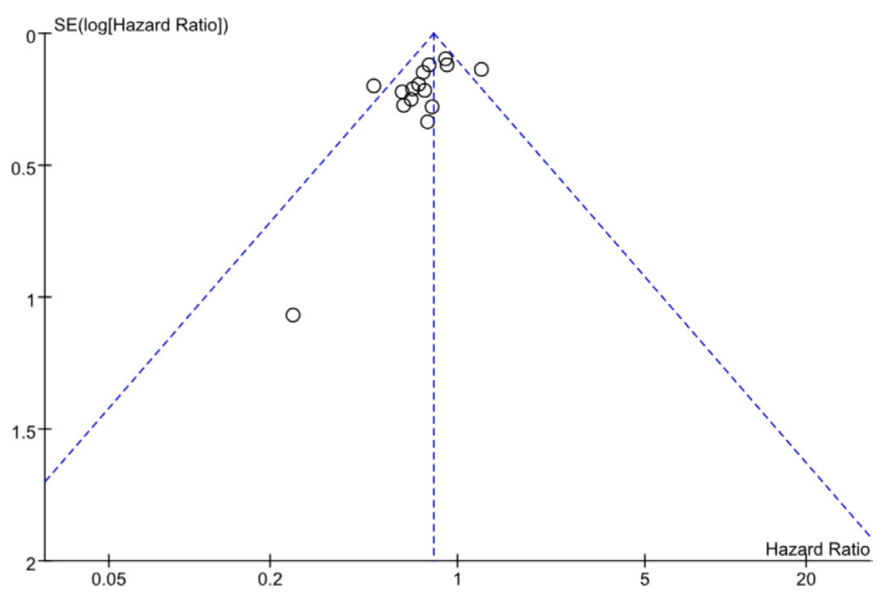

Figure 4 Funnel plot of publication bias.

cetuximab did not significantly increase the OS, which was consistent with the data from a previous report. ${ }^{48}$ It has been suggested that the reduced efficacy of cetuximab in combination with CRT is due to proinflammatory and antitumour proliferative effects of this mAb. ${ }^{49}$ Moreover, cetuximab may limit the free radical-induced DNA damage by platinum drugs, ${ }^{50}$ which could also explain the non-significant outcomes in this meta-analysis. For gefitinib, no improvement in OS was observed either, which was consistent with the results of a previous metaanalysis. ${ }^{51}$ Currently, there is no evidence that gefitinib can suppress EGFR, which indicates its limited clinical efficacy.

In this meta-analysis, there was no significant improvement in the PFS outcome in the anti-EGFR arm, which was consistent with the results of a previous meta-analysis. ${ }^{52} \mathrm{~A}$ longer PFS was only confirmed for nimotuzumab combination therapy, as also reported by Jing et $a l_{.}^{53}$ The biological characteristics of nimotuzumab differ from those of other anti-EGFR agents, which might explain its different clinical effects.

With regard to the ORR and DCR, significant differences were noted between the anti-EGFR and non-antiEGFR arms. Both anti-EGFR mAbs and TKIs, especially nimotuzumab, cetuximab and erlotinib, caused significant differences in the ORR and DCR for OC. However, previous studies have reported different ORRs and DCRs. ${ }^{54}$

Regarding the ORR, our results were consistent with those of some studies that indicated a significant advantage of combining anti-EGFR agents with CT, CRT or RT. $^{55} 56$ Furthermore, an ORR of $47.8 \%$ has been previously reported in the nimotuzumab group compared with $15.4 \%$ in the control group. ${ }^{17}$ Additionally, antiEGFR TKIs have been found to be superior to conventional therapy in terms of the ORR. ${ }^{57}$ Our meta-analysis indicated that erlotinib significantly increased the ORR. However, no combined data on the ORR have been reported for OC. It remains unknown whether erlotinib therapy is better than other treatment strategies owing to insufficient and conflicting homogeneity studies.
The DCR was greatly improved when anti-EGFR agents were combined with CT or RT; however, this result could not be confirmed as only few studies reported this outcome. Erlotinib and cetuximab demonstrated notable beneficial effects on the DCR in OC, which was consistent with the results of previous studies. ${ }^{485}$ Most meta-analyses have only reported results for $\mathrm{mAb}$ and TKI therapy, without specifying the individual drugs; therefore, it was impossible to verify the effectiveness of each drug.

This meta-analysis confirmed that patients with OC had higher incidences of myelosuppression, diarrhoea, acnelike rashes and hypomagnesaemia in the anti-EGFR than in the non-anti-EGFR arm, which was consistent with the data of previous studies. ${ }^{5859}$ An increased risk of myelosuppression was confirmed for combination therapy with anti-EGFR agents and CT. In particular, this meta-analysis showed that nimotuzumab increased the risk of myelosuppression. This finding might be due to the ability of nimotuzumab to enhance the sensitivity of tumour cells to $\mathrm{CT}^{43}$ However, these findings should be interpreted with caution because of the lack of sufficient high-quality, multicentre, large-sample RCTs.

Non-haematological toxicities, such as diarrhoea, acnelike rashes and hypomagnesaemia, occurred more often in patients with OC who received anti-EGFR agents with CRT than in those who received CRT alone. Furthermore, the combination of cetuximab or gefitinib with conventional treatment resulted in a higher risk of diarrhoea than conventional treatment alone. The underlying mechanisms of diarrhoea-causing effects of anti-EGFR mAbs and TKIs remain poorly understood and might be due to multiple factors, including altered gut motility, colonic crypt damage, changes to the intestinal microbiota and altered colonic transport. ${ }^{60}$ A plausible explanation could be the inhibition of the EGFR pathway in enterocytes. ${ }^{61}$ Thus, a better understanding of the underlying mechanisms may help in the identification of new approaches to the prophylaxis, risk reduction and development of more effective management of diarrhoea.

Acne-like rash is the most common side effect of antiEGFR agents, ${ }^{62}$ and this meta-analysis confirmed that antiEGFR mAbs and TKIs, such as cetuximab and erlotinib, could induce acne-like rashes. The mechanism leading to skin toxicity is not well known but undoubtedly involves the activation of associated signalling pathways, particularly the RAS/RAF/MEK/ERK pathway, which affects cell cycle regulation, including the proliferation and differentiation of epidermal cells. ${ }^{63}$ Skin toxicity often leads to physical and psychological discomfort, which may result in treatment interruptions, dose modifications or even non-adherence to anticancer therapies. ${ }^{62}$ Therefore, it is imperative to understand skin toxicities and develop proper approaches to control or relieve their adverse effects in patients.

An increased incidence of hypomagnesaemia was confirmed for cetuximab, in accordance with the data of another report. ${ }^{48}$ Hypomagnesaemia, a frequent side effect of cetuximab, is a serious electrolyte abnormality 
associated with an increased risk of seizures and cardiac events. ${ }^{64}$ The exact mechanism of cetuximab-induced hypomagnesaemia has not been well elucidated. Some studies have indicated that EGFR is strongly expressed in the kidney, particularly in the ascending limb of the loop of Henle, where $70 \%$ of the filtered magnesium is reabsorbed, and cetuximab might interfere with the magnesium transport by blocking EGFR. ${ }^{64}{ }^{65}$ However, hypomagnesaemia is often overlooked because of its nonspecific symptoms, which are attributed to underlying tumours or previous CT regimens ${ }^{66}$ Consequently, serum magnesium should be monitored during cetuximabbased therapy.

Several limitations should be considered when interpreting the results of this meta-analysis. First, we lacked access to individual patient data, which might have caused publication, reporting or selection bias. Second, because of the insufficient number of studies conducted, different anti-EGFR agents and conventional treatments were included in this meta-analysis, which increased clinical heterogeneity of the data. Subsequently, subgroup and sensitivity analyses were performed to improve the reliability of our results. Third, the methods were unclear, especially in the domain of 'allocation concealment and blinding', which might have affected the overall methodological quality of the included studies. High methodological quality of RCTs is needed for further research. Finally, considering all these limitations, the results of this meta-analysis should be interpreted with caution, and further research is necessary to verify the effects of antiEGFR therapy in OC.

In conclusion, this meta-analysis indicated that the addition of anti-EGFR agents to multimodal therapy was beneficial for patients with OC in terms of their OS, ORR and DCR. Therefore, anti-EGFR agents may be recommended as a treatment option for OC to improve treatment outcomes. The results of this study may help establish an optimum therapeutic regimen for OC. However, considering the higher incidences of myelosuppression, diarrhoea, acne-like rashes and hypomagnesaemia when using the combination of anti-EGFR agents and multimodal therapy, careful monitoring of patients and effective management of their condition are recommended. Further high-quality, large-scale, multicentre studies are needed to confirm the efficacy and safety of anti-EGFR agents in patients with OC.

\section{Author affiliations}

${ }^{1}$ School of Nursing, Liaoning University of Traditional Chinese Medicine, Shenyang, China

${ }^{2}$ School of Nursing, Tianjin Medical University, Tianjin, China

${ }^{3}$ Department of Colorectal Surgery, Liaoning Cancer Institute and Hospital,

Shenyang, China

${ }^{4}$ Department of Critical Care Medicine, Liaoning Cancer Institute and Hospital, Shenyang, China

${ }^{5}$ Department of Science and Technology, Liaoning University of Traditional Chinese Medicine, Shenyang, China

Contributors LZ and RY designed the study; LZ, YS and NJ performed the literature search, extracted the data and evaluated the quality of included studies;
LZ, YH, BD, WL, YH, YC and HL conducted statistical analyses; LZ, YS and NJ were involved in the interpretation of the results; LZ, YS, NJ and RY were responsible for the writing and critical revision of the manuscript. All authors read and approved the final manuscript.

Funding This work was supported by the Liaoning Social Science Planning Fund Project (grant number L19CSH002).

Competing interests None declared.

Patient consent for publication Not required.

Provenance and peer review Not commissioned; externally peer reviewed.

Data availability statement All data relevant to the study are included in the article or uploaded as supplementary information.

Supplemental material This content has been supplied by the author(s). It has not been vetted by BMJ Publishing Group Limited (BMJ) and may not have been peer-reviewed. Any opinions or recommendations discussed are solely those of the author(s) and are not endorsed by BMJ. BMJ disclaims all liability and responsibility arising from any reliance placed on the content. Where the content includes any translated material, BMJ does not warrant the accuracy and reliability of the translations (including but not limited to local regulations, clinical guidelines, terminology, drug names and drug dosages), and is not responsible for any error and/or omissions arising from translation and adaptation or otherwise.

Open access This is an open access article distributed in accordance with the Creative Commons Attribution Non Commercial (CC BY-NC 4.0) license, which permits others to distribute, remix, adapt, build upon this work non-commercially, and license their derivative works on different terms, provided the original work is properly cited, appropriate credit is given, any changes made indicated, and the use is non-commercial. See: http://creativecommons.org/licenses/by-nc/4.0/.

\section{ORCID iD}

Rui Yu http://orcid.org/0000-0002-4506-5081

\section{REFERENCES}

1 Bray F, Ferlay J, Soerjomataram I, et al. Global cancer statistics 2018: GLOBOCAN estimates of incidence and mortality worldwide for 36 cancers in 185 countries. CA Cancer J Clin 2018;68:394-424.

2 Chen W, Zheng R, Baade PD, et al. Cancer statistics in China, 2015. CA Cancer J Clin 2016;66:115-32.

3 Pennathur A, Gibson MK, Jobe BA, et al. Oesophageal carcinoma. Lancet 2013;381:400-12.

4 Sudo K, Xiao L, Wadhwa R, et al. Importance of surveillance and success of salvage strategies after definitive chemoradiation in patients with esophageal cancer. J Clin Oncol 2014;32:3400-5.

5 Conroy T, Galais M-P, Raoul J-L, et al. Definitive chemoradiotherapy with FOLFOX versus fluorouracil and cisplatin in patients with oesophageal cancer (PRODIGE5/ACCORD17): final results of a randomised, phase 2/3 trial. Lancet Oncol 2014;15:305-14.

6 Kelsen DP, Winter KA, Gunderson LL, et al. Long-Term results of RTOG trial 8911 (USA intergroup 113): a random assignment trial comparison of chemotherapy followed by surgery compared with surgery alone for esophageal cancer. J Clin Oncol 2007;25:3719-25.

7 Minsky BD, Pajak TF, Ginsberg RJ, et al. Int 0123 (radiation therapy Oncology Group 94-05) phase III trial of combined-modality therapy for esophageal cancer: high-dose versus standard-dose radiation therapy. J Clin Oncol 2002;20:1167-74.

8 Minsky BD, Neuberg D, Kelsen DP, et al. Final report of intergroup trial 0122 (ECoG PE-289, RTOG 90-12): phase II trial of neoadjuvant chemotherapy plus concurrent chemotherapy and high-dose radiation for squamous cell carcinoma of the esophagus. Int $\mathrm{J}$ Radiat Oncol Biol Phys 1999;43:517-23.

9 Mendelsohn $\mathrm{J}$. The epidermal growth factor receptor as a target for cancer therapy. Endocr Relat Cancer 2001;8:3-9.

10 Zhang W, Zhu H, Liu X, et al. Epidermal growth factor receptor is a prognosis predictor in patients with esophageal squamous cell carcinoma. Ann Thorac Surg 2014;98:513-9.

11 Lorenzen S, Schuster T, Porschen R, et al. Cetuximab plus cisplatin-5-fluorouracil versus cisplatin-5-fluorouracil alone in first-line metastatic squamous cell carcinoma of the esophagus: a randomized phase II study of the Arbeitsgemeinschaft Internistische Onkologie. Ann Oncol 2009;20:1667-73.

12 Lammering G, Hewit TH, Hawkins WT, et al. Epidermal growth factor receptor as a genetic therapy target for carcinoma cell radiosensitization. J Natl Cancer Inst 2001;93:921-9. 
13 Yewale C, Baradia D, Vhora I, et al. Epidermal growth factor receptor targeting in cancer: a review of trends and strategies. Biomaterials 2013;34:8690-707

14 Ruhstaller T, Thuss-Patience P, Hayoz S, et al. Neoadjuvant chemotherapy followed by chemoradiation and surgery with and without cetuximab in patients with resectable esophageal cancer: a randomized, open-label, phase III trial (SAKK 75/08). Ann Oncol 2018;29:1386-93.

15 Suntharalingam M, Winter K, Ilson D, et al. Effect of the addition of cetuximab to paclitaxel, cisplatin, and radiation therapy for patients with esophageal cancer: the NRG oncology RTOG 0436 phase 3 randomized clinical trial. JAMA Oncol 2017;3:1520-8.

16 de Castro Junior G, Segalla JG, de Azevedo SJ, et al. A randomised phase II study of chemoradiotherapy with or without Nimotuzumab in locally advanced oesophageal cancer: NICE trial. Eur J Cancer 2018;88:21-30

17 Ramos-Suzarte M, Lorenzo-Luaces P, Lazo NG, et al. Treatment of malignant, non-resectable, epithelial origin esophageal tumours with the humanized anti-epidermal growth factor antibody Nimotuzumab combined with radiation therapy and chemotherapy. Cancer Biol Ther 2012;13:600-5.

18 Kordes S, van Berge Henegouwen MI, Hulshof MC, et al. Preoperative chemoradiation therapy in combination with panitumumab for patients with resectable esophageal cancer: the PACT study. Int J Radiat Oncol Biol Phys 2014;90:190-6.

19 Wu S-X, Wang L-H, Luo H-L, et al. Randomised phase III trial of concurrent chemoradiotherapy with extended nodal irradiation and erlotinib in patients with inoperable oesophageal squamous cell cancer. Eur J Cancer 2018;93:99-107.

20 Chen YD, XN L, Shao ZF. Clinical observation of Tarceva in combination with chemo-therapy for patients with esophageal cancer after postoperative treatment. Modern Hos-pital 2014;14:44-6.

21 Zeng $\mathrm{H}$, Huang XQ, Song WL. Clinical observation of second line treatment of gefltinib combined with oxaliplatin and fluorouracil for advanced esophageal cancer. Chin J Clin Oncol 2014:325-7.

22 Dutton SJ, Ferry DR, Blazeby JM, et al. Gefitinib for oesophageal cancer progressing after chemotherapy (COG): a phase 3 , multicentre, double-blind, placebo-controlled randomised trial. Lancet Oncol 2014;15:894-904.

23 Rice TW, Ishwaran H, Ferguson MK, et al. Cancer of the esophagus and esophagogastric junction: an eighth edition staging primer. $J$ Thorac Oncol 2017;12:36-42.

24 Tierney JF, Stewart LA, Ghersi D, et al. Practical methods for incorporating summary time-to-event data into meta-analysis. Trials 2007;8:1-16.

25 Chen YD, Wang YD, XN L. Efficacy of cetuximab combined with chemotherapy in postoperative adjuvant treatment of advanced esophageal cancer. J Prac Med 2014;30:2862-3.

26 Sun CJ, Chen Y. The clinical research of Nimotuzumab combined with radiotherapy and chemotherapy for advanced esophageal cancer. J Clin Med Liter 2014;1:2346-8.

27 Wu CR, Zhang QZ, Yang W. Clinical study on Nimotuzumab combined with intensity- modulated radiotherapy and chemotherapy for esophageal cancer. J Chin Oncol 2014;20:500-3.

28 Moher D, Liberati A, Tetzlaff J, et al. Preferred reporting items for systematic reviews and meta-analyses: the PRISMA statement. BMJ 2009;339:b2535.

29 Fu GL. Curative effect of Nimotuzumab combined with concurrent chemoradiotherapy in the treatment of locally advanced esophageal carcinoma. Chin J Prim Med Pharm 2017;24:3457-61.

30 Chen YL, SP L, Lian YN. Clinical study on Nimotuzumab combined with nadaplatin concurrent chemoradiotherapy for local advanced esophageal carcinoma. J North Pharm 2016;13:140-1.

31 Zhao HN. Effect of erlotinib combined with radiotherapy in the treatment of esophageal cancer. Health Guide 2016;22:231-2.

$32 \mathrm{Ye} \mathrm{HX}$. Clinical study of concurrent chemoradiotherapy with erlotinib in esophageal cancer. China Prac Med 2015;10:174-6.

33 Luo HT, Liang CX. Effect of intensity-modulated radiotherapy combined with docetaxel and Nimotuzumab in treatment of patients with esophageal carcinoma. Modern Oncol 2015;23:219-22.

34 Zhang P. Efficacy of radiotherapy combined with targeted drugs in the treatment of patients with esophageal cancer. China Prac Med 2014;9:115-6.

35 Shi YR, Wang $H$, Yang JR. Clinical study on late course accelerated hyperfractionated intensity-modulated radiation therapy plus Nimotuzumab for esophageal carcinoma. Cancer Res and Clinic 2014;26:514-7.

$36 \mathrm{Xu} \mathrm{Z}$, Chen J, Tao M. Exploratory study on the treatment of advanced esophageal squamous cell carcinoma with Nimotuzumab and PF regimen. J Pracl Oncol 2013;28:650-3.
37 Lu ZG. Auxiliary effect of cetuximab in chemotherapy for intermediate and advanced esophageal cancer. Guangxi Med J 2017;39:1015-7

38 Zhou M, Sun TT, Wang ZZ. Study of targeted therapy of radiotherapy combined with chemotherapy in treatment of advanced esophageal carcinoma. Modern Med J 2015;43:740-3.

39 Chen $\mathrm{H}$. Observation on efficacy of erlotinib hydrochloride tablets combined with taxol and cisplatin of conformal radiotherapy in treatment of esophageal cancer. Evaluation and analysis of drug-use in hospitals of China 2016;16:476-8.

40 Crosby T, Hurt CN, Falk S, et al. Long-Term results and recurrence patterns from SCOPE-1: a phase II/III randomised trial of definitive chemoradiotherapy +/- cetuximab in oesophageal cancer. $\mathrm{Br} J$ Cancer 2017;116:709-16.

41 Crosby T, Hurt CN, Falk S, et al. Chemoradiotherapy with or without cetuximab in patients with oesophageal cancer (SCOPE1): a multicentre, phase $2 / 3$ randomised trial. Lancet Oncol 2013;14:627-37.

42 Crombet-Ramos T, Rak J, Pérez R, et al. Antiproliferative, antiangiogenic and proapoptotic activity of h-R3: a humanized antiEGFR antibody. Int J Cancer 2002;101:567-75.

43 Song H, Pan B, Yi J, et al. Featured article: autophagic activation with Nimotuzumab enhanced chemosensitivity and radiosensitivity of esophageal squamous cell carcinoma. Exp Biol Med 2014;239:529-41.

44 Höpfner M, Sutter AP, Huether A, et al. Targeting the epidermal growth factor receptor by gefitinib for treatment of hepatocellular carcinoma. J Hepatol 2004;41:1008-16.

45 Höpfner M, Sutter AP, Gerst B, et al. A novel approach in the treatment of neuroendocrine gastrointestinal tumours. targeting the epidermal growth factor receptor by gefitinib (ZD1839). Br J Cancer 2003;89:1766-75.

46 Huang S-M, Li J, Armstrong EA, et al. Modulation of radiation response and tumor-induced angiogenesis after epidermal growth factor receptor inhibition by ZD1839 (Iressa). Cancer Res 2002;62:4300-6.

47 Sutter AP, Höpfner M, Huether A, et al. Targeting the epidermal growth factor receptor by erlotinib (Tarceva) for the treatment of esophageal cancer. Int J Cancer 2006;118:1814-22.

48 Huang Z-H, Ma X-W, Zhang J, et al. Cetuximab for esophageal cancer: an updated meta-analysis of randomized controlled trials. BMC Cancer 2018;18:1170.

49 Glynne-Jones R, Mawdsley S, Harrison M. Cetuximab and chemoradiation for rectal cancer--is the water getting muddy? Acta Oncol 2010;49:278-86.

50 Dahan L, Sadok A, Formento J-L, et al. Modulation of cellular redox state underlies antagonism between oxaliplatin and cetuximab in human colorectal cancer cell lines. Br J Pharmacol 2009:158:610-20.

51 Ku GY, Haaland BA, de Lima Lopes G. Gefitinib vs. chemotherapy as first-line therapy in advanced non-small cell lung cancer: metaanalysis of phase III trials. Lung Cancer 2011;74:469-73.

52 Janmaat VT, Steyerberg EW, van der Gaast A, et al. Palliative chemotherapy and targeted therapies for esophageal and gastroesophageal junction cancer. Cochrane Database Syst Rev 2017;11:D4063.

53 Jing W, Yan W, Liu Y, et al. Slight advantages of Nimotuzumab versus cetuximab plus concurrent chemoradiotherapy in locally advanced esophageal squamous cell carcinoma. Cancer Biol Ther 2019;20:1121-6.

54 Tian Y, Lin J, Tian Y, et al. Efficacy and safety of anti-EGFR agents administered concurrently with standard therapies for patients with head and neck squamous cell carcinoma: a systematic review and meta-analysis of randomized controlled trials. Int J Cancer 2018;142:2198-206.

55 Pietrantonio F, Cremolini C, Petrelli F, et al. First-Line anti-EGFR monoclonal antibodies in panRAS wild-type metastatic colorectal cancer: a systematic review and meta-analysis. Crit Rev Oncol Hematol 2015;96:156-66.

56 Luo H-Q, Han L, Jiang Y. Meta-Analysis of six randomized control trials of chemotherapy plus anti-HER monoclonal antibody for advanced gastric and gastroesophageal cancer. Asian Pac J Cancer Prev 2014;15:5343-8.

57 Zhang Y, Sheng J, Yang Y, et al. Optimized selection of three major EGFR-TKIs in advanced EGFR-positive non-small cell lung cancer: a network meta-analysis. Oncotarget 2016;7:20093-108.

58 Sandler AB. Nondermatologic adverse events associated with antiEGFR therapy. Oncology 2006;20:35-40.

59 Han X, Lu N, Pan Y, et al. Nimotuzumab combined with chemotherapy is a promising treatment for locally advanced and metastatic esophageal cancer. Med Sci Monit 2017;23:412-8. 
$60 \mathrm{Li} \mathrm{J}, \mathrm{Gu}$ J. Diarrhea with epidermal growth factor receptor tyrosine kinase inhibitors in cancer patients: a meta-analysis of randomized controlled trials. Crit Rev Oncol Hematol 2019;134:31-8.

61 Miroddi M, Sterrantino C, Simonelli I, et al. Risk of grade 3-4 diarrhea and mucositis in colorectal cancer patients receiving anti-EGFR monoclonal antibodies regimens: a meta-analysis of 18 randomized controlled clinical trials. Crit Rev Oncol Hematol 2015;96:355-71.

62 Peng Y, Li Q, Zhang J, et al. Update review of skin adverse events during treatment of lung cancer and colorectal carcinoma with epidermal growth receptor factor inhibitors. Biosci Trends 2019;12:537-52.
63 Manousaridis I, Mavridou S, Goerdt S, et al. Cutaneous side effects of inhibitors of the Ras/Raf/MEK/ERK signalling pathway and their management. J Eur Acad Dermatol Venereol 2013;27:11-18.

64 Fakih MG, Wilding G, Lombardo J. Cetuximab-induced hypomagnesemia in patients with colorectal cancer. Clin Colorectal Cancer 2006;6:152-6.

65 Cao Y, Liao C, Tan A, et al. Meta-Analysis of incidence and risk of hypomagnesemia with cetuximab for advanced cancer. Chemotherapy 2010;56:459-65.

66 Schrag D, Chung KY, Flombaum C, et al. Cetuximab therapy and symptomatic hypomagnesemia. J Natl Cancer Inst 2005;97:1221-4. 